\title{
DesarRollo de especies leñosas nativas y exóticas en CIPRESALES PATAGÓNICOS
}

\author{
MARINA STECCONI',2, LAURA QUEVEDO'1, AMARU MAGNIN'1, CRISTIAN TORRES ${ }^{1}$, \\ KAREN LEDIUK ${ }^{1}$, MAYA SVRIZ1 ${ }^{1}$, JAVIER GROSFELD ${ }^{3}$ y JAVIER PUNTIERI ${ }^{1,2}$
}

\begin{abstract}
Summary: Development of native and exotic woody species in the Patagonian cypress forests. The growth and architecture of forest-dwelling woody plants affect the dynamics of these communities. The present study was aimed at improving our understanding of the dynamics of Argentinean Austrocedrus chilensis forests through the study of length growth and architecture of some of the most frequent native and exotic woody species in these communities (natives: Aristotelia chilensis, Berberis microphylla, Lomatia hirsuta, Maytenus boaria, Myoschilos oblongum and Schinus patagonicus; exotics: Juniperus communis, Prunus avium, Pseudotsuga menziesii and Rosa rubiginosa). During the study period (20122014), the length growth of the main axis was not necessarily related to the height growth of labeled plants. Reductions in height were found for individuals of all species but $P$. menziesii and $P$. avium, which are exotics in Patagonia. The majority of plants presented notable deviations of the main axis from the vertical; the extent of this deviation was negatively correlated with both the length of the distal main-axis shoot and the height variation of the plant. The species under survey may be categorized in four functional groups according to their architecture. The invasion of these cypress forests by exotic species such as $P$. menziesii y $P$. avium, which belong to functional groups different from those of the most frequent coexisting native species, may promote the competitive replacement of native species by exotic species.
\end{abstract}

Key words: Annual shoot, architecture, functional groups, invasions, main axis, reiteration.

Resumen: El crecimiento y la arquitectura de las especies leñosas que habitan los bosques inciden en la dinámica de estas comunidades. El objetivo principal de este estudio fue mejorar la comprensión de la dinámica de los cipresales patagónicos a partir del análisis del crecimiento longitudinal y de la arquitectura de las especies leñosas más abundantes en esas comunidades, las nativas: Aristotelia chilensis, Berberis microphylla, Lomatia hirsuta, Maytenus boaria, Myoschilos oblongum y Schinus patagonicus; y las exóticas: Juniperus communis, Prunus avium, Pseudotsuga menziesii y Rosa rubiginosa. El crecimiento longitudinal del eje principal no se relacionó necesariamente con el aumento en altura de las plantas durante el período de estudio (2012-14). Se registraron decrecimientos en altura en todas las especies excepto en $P$. menziesii y $P$. avium, exóticas para Patagonia. La mayoría de los individuos presentaron notables desviaciones de su eje principal de la vertical, las que se correlacionaron negativamente con la longitud del brote distal y con la variación en altura de las plantas. Se agruparon las especies en cuatro grupos funcionales, de acuerdo a su arquitectura y crecimiento. La invasión de los cipresales por parte de especies exóticas como $P$. menziesii y $P$. avium, asociadas a grupos funcionales distintos a los de las especies nativas más frecuentes, podría impactar significativamente en la dinámica de esas comunidades promoviendo el reemplazo de las especies nativas por las exóticas dominantes.

Palabras clave: Brote anual, arquitectura, grupos funcionales, invasiones, eje principal, reiteración.

\footnotetext{
${ }^{1}$ INIBIOMA (Universidad Nacional del Comahue - CONICET), Quintral 1250, San Carlos de Bariloche.

${ }^{2}$ Universidad Nacional de Río Negro, Sede Andina.

${ }^{3}$ Centro Científico y Tecnológico Patagonia Norte, CONICET, San Carlos de Bariloche.
} 


\section{INTRODUCCIÓN}

Cada una de las especies leñosas que conviven en un bosque posee atributos morfológicos, fisiológicos y fenológicos que la asocian a un determinado grupo funcional, por vincularse tales atributos a las respuestas de la especie a las condiciones ambientales e incidir en las interacciones intra- e inter-específicas (Reich et al., 1998; Diaz et al., 1999; Cornelissen et al., 2003; Díaz et al., 2016). Por ejemplo, en una comunidad boscosa pueden habitar especies que favorecen la regeneración de otras (Connell \& Slatyer, 1977; Henríquez \& Lusk, 2005; Lediuk et al., 2014) y especies que incrementan la probabilidad de generación y propagación de incendios (Kitzberger et al., 1997; LaRade \& Bork, 2011). Dentro de este esquema, las especies exóticas pueden ocupar "nichos vacantes" dentro de la comunidad o asemejarse a las especies nativas respecto de sus grupos funcionales, compitiendo con éstas en mayor o menor medida por los recursos disponibles. Estas diferencias y similitudes entre las especies que coexisten en un bosque pueden ser fundamentales en el inicio de nuevas invasiones por parte de las especies exóticas (Lockwood et al., 2007; Wolkovich \& Cleland, 2011; Lediuk et al., 2014, 2016).

En la última década se ha reconocido que la identificación de grupos funcionales de las especies que coexisten en los bosques aporta significativamente en la comprensión de la dinámica de esas comunidades y la predicción de su desarrollo (e.g. Poorter et al., 2006; Díaz et al., 1999, 2016). Hasta el momento, los estudios que proponen la clasificación de las plantas en grupos funcionales han incorporado pocas características arquitecturales (Reich et al., 2003; Cornelissen et al., 2003; Pérez-Harguindeguy et al., 2013). Entre estas características se encuentra la formación de un tronco vertical bien diferenciado de sus ramas, un atributo que puede condicionar la abundancia de esa especie al incidir en sus relaciones de competencia con otras especies por el recurso lumínico (Givnish, 1988; Canham, 1988; Millet et al., 1998; Vester, 2002; Nagashima \& Hikosaka, 2011; Ford, 2014). Dado que el grado de diferenciación del tronco respecto de sus ejes laterales puede variar entre plantas de la misma especie debido a factores ontogenéticos y/o ambientales (Edelin, 1991; Barthélémy \& Caraglio, 2007; Stecconi et al., 2010; Hérault et al., 2011), el registro de la diferenciación del tronco a partir de la observación de plantas en un único momento puede no ser suficiente para determinar el grupo funcional de la especie (Millet et al., 1998; Stecconi et al., 2014). Por ejemplo, dos especies de sotobosque que desarrollan un tronco vertical dominante en fases ontogenéticas tempranas, pueden diferenciarse entre sí con posterioridad si una de ellas conserva esa arquitectura, pudiendo acercarse al dosel del bosque, y la otra no. Es posible lograr una mejor comprensión de los cambios en las relaciones inter-específicas y en la estructura de la comunidad a lo largo del tiempo a partir del seguimiento del desarrollo de individuos marcados (e.g. Hallé et al., 1978; Aguiar \& Sala, 1994; Rodríguez-García et al., 2011; Zalamea et al., 2012). El conocimiento del desarrollo de las especies de sotobosque desde la perspectiva arquitectural puede aportar herramientas en el manejo sustentable del bosque y el resguardo de los servicios ecosistémicos (Sekercioglu, 2010).

Los bosques de ciprés de la cordillera, Austrocedrus chilensis (D. Don) Pic. Ser. et Biz. (Cupressaceae; en adelante denominado ciprés) ocupan unas 141.000 ha en Argentina (Bran et al., 2002; Pastorino \& Gallo, 2009) y 45.000 ha en Chile (CONAF 1997 en Pastorino \& Gallo, 2009). En Argentina estos bosques abarcan un amplio rango del gradiente E-O de precipitación, desde la estepa, con $600 \mathrm{~mm}$ anuales, hasta el bosque lluvioso, con más de $2500 \mathrm{~mm}$ anuales (Donoso, 2006). Los bosques compactos y monoespecíficos de ciprés se encuentran en la ecoregión Complejo Bosque de Transición Ciprés- Lenga, con niveles de precipitación entre 600 y $1700 \mathrm{~mm}$ anuales (Dezzotti \& Sancholuz, 1991; Morello et al., 2012).

Los bosques de ciprés, tanto en Argentina como en Chile, son afectados por actividades humanas, como la extracción de madera, los incendios y la presencia de ganado (Donoso, 2006; Tortorelli, 2009; Morello et al., 2012). Estas actividades disminuyen la tasa de regeneración de los cipresales, contribuyendo a su fragmentación y pérdida de diversidad (ver Carabelli et al., 2006). Varios estudios analizaron los efectos de disturbios y de las variaciones climáticas sobre la dinámica de los bosques de ciprés (Veblen et al., 1989, 1992; Kitzberger et al., 1997, 2000; Villalba \& Veblen, 1998; Letourneau et al., 2004; Amoroso et al., 2012). Se ha demostrado que algunas especies leñosas que habitan en estos bosques generan 
condiciones más favorables para el establecimiento del ciprés luego de disturbios (Gobbi \& Schlichter, 1998; Gyenge et al., 2007; Steinke et al., 2008; Núñez et al., 2009). Entre estas especies con función "facilitadora" se encuentran las nativas Schinus patagonicus (Phil.) I.M. Johnst. ex Cabrera, Maytenus boaria Mol., Lomatia hirsuta (Lam) Diels y Berberis microphylla G. Forst., y la exótica Rosa rubiginosa L. (Kitzberger et al., 2000; Svriz et al., 2013), que abundan en el estrato arbustivo de los bosques patagónicos, aunque las tres primeras pueden alcanzar porte arbóreo (Dimitri, 1978). Se ha documentado que los cipreses superan en altura a sus plantas facilitadoras, recomponiendo el dosel arbóreo, tras lo cual las plantas facilitadoras menos tolerantes a la sombra mueren (Letourneau et al., 2004; Núñez et al., 2009). Por otro lado, no se conocen registros sistemáticos sobre el crecimiento de los arbustos bajo la cobertura de ciprés y sobre sus posibles efectos en el desarrollo de esos bosques. En el presente estudio se analizan las variaciones temporales del tamaño y arquitectura, y la fenología de las plantas leñosas que habitan bajo el dosel del ciprés con el objetivo de caracterizar grupos funcionales y aportar al conocimiento de la dinámica de estos bosques.

\section{Materiales y Métodos}

Sitios de muestreo

Se delimitaron cuatro parcelas de $50 \times 50 \mathrm{~m}$ en bosques de ciprés compactos (parcelas I-IV, Tabla 1). Las parcelas I y II fueron delimitadas en noviembre y diciembre de 2011 en el paraje Las Golondrinas, provincia de Chubut (Lotes 9 y 15, bajo jurisdicción del INTA Bariloche) y en Cascada Escondida, Mallín Ahogado, provincia de Río Negro (INTA Bariloche), respectivamente. Las parcelas III y IV fueron delimitadas en septiembre de 2012 en la Estancia Siete Cóndores, Confluencia Traful, provincia de Neuquén, separadas entre sí por una distancia de alrededor de $200 \mathrm{~m}$. Para las cuatro parcelas existen registros de incendios y presencia de ganado y ciervos exóticos desde la primera mitad del siglo XX (Veblen \& Lorenz, 1988; Letourneau, 2006).

La abundancia de las plantas leñosas se estimó mediante la cobertura aérea medida por proyección sobre cinco líneas de $50 \mathrm{~m}$ cada una distribuidas al azar a través del método línea-intercepción, hasta los $3 \mathrm{~m}$ de altura (Mueller-Dombois \& Ellenberg, 1974). Las especies leñosas del sotobosque con mayor cobertura aérea fueron las nativas: Aristotelia chilensis (Mol.) Stuntz, Berberis microphylla, Lomatia hirsuta, Maytenus boaria, Myoschilos oblongum Ruiz \& Pav. y Schinus patagonicus; y las exóticas: Juniperus communis L., Prunus avium L., Pseudotsuga menziesii (Mirb.) Franco y Rosa rubiginosa (Tabla 2). Para cada una de estas especies se seleccionaron al azar, en cada parcela, entre cinco y diez individuos, que fueron marcados para su seguimiento en el tiempo. Además, sobre más de 50 individuos no marcados de cada una de esas especies se realizaron observaciones fenológicas y morfológicas. La edad del eje principal de los individuos, obtenida en forma no destructiva (ver abajo) varió entre 2 y 28 años (Tabla 2).

Tabla 1. Localización de las parcelas de muestreo donde se midieron las plantas marcadas.

\begin{tabular}{|lccccc|}
\hline \multicolumn{1}{|c}{ Sitio } & Parcela & $\begin{array}{c}\text { Latitud } \\
\left({ }^{\circ} \mathbf{S}\right)\end{array}$ & $\begin{array}{c}\text { Longitud } \\
\left({ }^{\circ} \mathrm{O}\right)\end{array}$ & $\begin{array}{c}\text { Altitud } \\
\text { (m s.n.m.) }\end{array}$ & $\begin{array}{c}\text { Precipitación } \\
\text { (mm/año) }\end{array}$ \\
\hline Las Golondrinas & I & $42^{\circ} 00^{\prime} 22^{\prime \prime}$ & $71^{\circ} 32^{\prime} 18^{\prime \prime}$ & 398 & $807,2^{\text {a }}$ \\
Mallín Ahogado & II & $41^{\circ} 55^{\prime} 28^{\prime \prime}$ & $71^{\circ} 32^{\prime} 23^{\prime \prime}$ & 454 & $1149,8^{\mathrm{b}}$ \\
Confluencia & III & $40^{\circ} 45^{\prime} 03^{\prime \prime}$ & $71^{\circ} 07^{\prime} 13^{\prime \prime}$ & 780 & $800-1000^{\circ}$ \\
Confluencia & IV & $40^{\circ} 45^{\prime} 02^{\prime \prime}$ & $71^{\circ} 07^{\prime} 08^{\prime \prime}$ & 783 & $800-1000^{\circ}$ \\
\hline
\end{tabular}

a Letourneau et al., 2016, b Lebed O., comunicación personal, c Barros et al., 1983. 
Tabla 2. Características de las especies leñosas incluidas en las parcelas del presente estudio. Se indica el origen ( $\mathrm{Or}^{2}$, n: nativa, e: exótica), persistencia de las Hojas ${ }^{3}$ (C: caduca, P: perenne, SC: semicaduca), presencia de Reiteraciones basales ${ }^{4}$ (+: presencia, -: ausencia), Altura ${ }^{5}$ de las plantas según bibliografía, $\mathrm{N}$ : tamaño de la muestra.

\begin{tabular}{|lcccccccc|}
\hline \multicolumn{1}{|c}{ Especie $^{1}$} & Or $^{2}$ & Hojas $^{3}$ & $\begin{array}{c}\text { Reit. } \\
\text { basales }^{4}\end{array}$ & Altura $^{5}$ (m) & Parcela & N & Edad mín. Edad máx. \\
\hline Aristotelia chilensis & $\mathrm{n}$ & $\mathrm{SC}$ & + & 5 & I, II & 10 & 8 & 16 \\
Berberis microphylla & $\mathrm{n}$ & $\mathrm{P}$ & + & 2,5 & III, IV & 20 & 2 & 12 \\
Juniperus communis & $\mathrm{e}$ & $\mathrm{P}$ & - & 10 & I & 5 & 11 & 28 \\
Lomatia hirsuta & $\mathrm{n}$ & $\mathrm{P}$ & + & 15 & I, II, III, IV & 30 & 3 & 16 \\
Maytenus boaria & $\mathrm{n}$ & $\mathrm{P}$ & + & 15 & I, II & 5 & 7 & 18 \\
Myoschilos oblongum & $\mathrm{n}$ & $\mathrm{C}$ & + & 2 & I, II & 5 & 8 & 13 \\
Prunus avium & $\mathrm{e}$ & $\mathrm{C}$ & - & 20 & I & 5 & 11 & 22 \\
Pseudotsuga menziesii & $\mathrm{e}$ & $\mathrm{P}$ & - & 90 & I, II & 10 & 8 & 14 \\
Rosa rubiginosa & $\mathrm{e}$ & $\mathrm{C}$ & + & 3 & I, II & 10 & 2 & 6 \\
Schinus patagonicus & $\mathrm{n}$ & $\mathrm{P}$ & + & 5 & I, II, III, IV & 30 & 3 & 16 \\
\hline
\end{tabular}

* La nomenclatura sigue el Catálogo de la Flora Argentina del Instituto Darwinion (HYPERLINK "http://www. floraargentina.edu.ar" www.floraargentina.edu.ar).

\section{Crecimiento}

Al inicio del estudio se rotuló, para cada planta, el eje principal (eje de mayor altura al momento de la medición; Fig. 1) y se midieron la altura y el diámetro basal del mismo al inicio y al final del estudio. Los tamaños iniciales se midieron en noviembre de 2011 en el caso de las plantas de las parcelas I y II, y en septiembre de 2012 para las plantas de las parcelas III y IV. Al final de la estación de crecimiento 2013-2014, para cada individuo marcado se registraron: la altura total, la longitud del segmento de tallo derivado del crecimiento primario del eje en esa estación (de aquí en adelante denominado brote anual) y la presencia de ramas desarrolladas a partir del brote anual medido (Fig. 1). La longitud y ramificación del brote anual producido por un eje son indicadores del crecimiento primario de ese eje y permiten realizar comparaciones entre plantas de la misma especie y de diferentes especies (Puntieri et al., 2003; Barthélémy \& Caraglio, 2007).

Con el fin de evaluar el grado de diferenciación del tronco de cada una de las plantas marcadas, en marzo de 2014 se registró la desviación del eje principal respecto de la vertical (DV; Fig. 1). Dicha estimación se obtuvo a partir de la medición de la distancia horizontal entre la proyección vertical del extremo distal del eje principal de la planta en la superficie del suelo y el punto de contacto del eje principal con el suelo. En ese momento se estimó la edad del eje principal de cada planta mediante

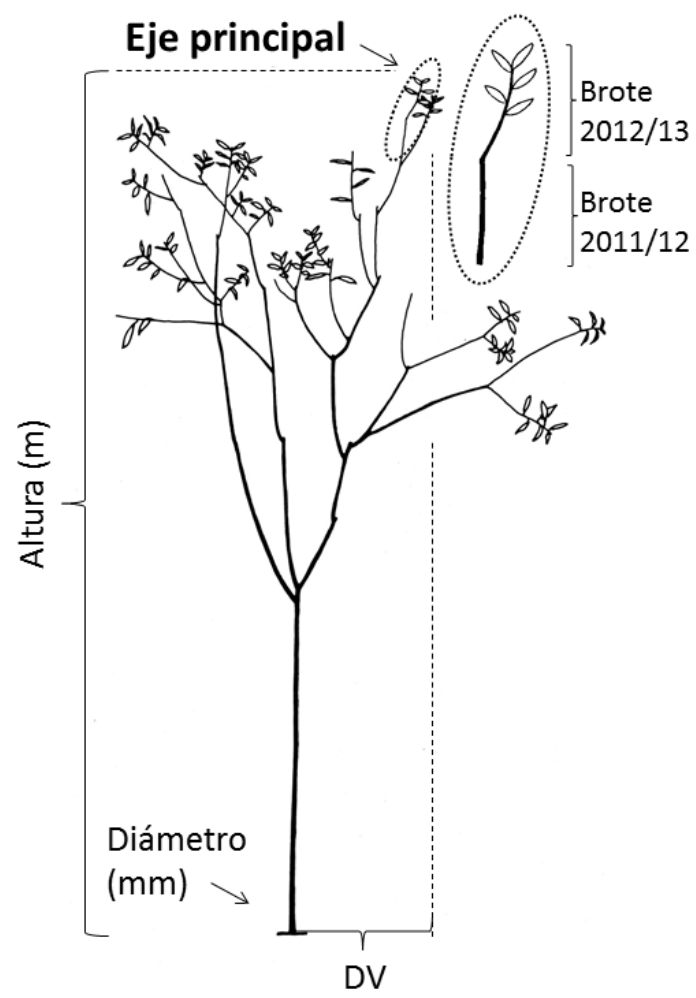

Fig. 1. Representación de un individuo de Maytenus boaria de alrededor de 14 años del estrato arbustivo de un cipresal. Se indican los dos brotes anuales más recientes del eje principal, la altura y el diámetro basal del eje principal, la desviación de la vertical (DV) del eje principal. 
el conteo de brotes anuales sucesivos, desde el nivel del suelo hasta el extremo distal. Para ello se emplearon marcadores morfológicos identificados a lo largo del primer año de muestreo: longitud de entrenudos, presencia de catafilos, posición de las ramas principales sobre el tronco y color de la superficie externa del tallo (Fig. 2; Barthélémy et al., 1999; Puntieri et al., 1999).

Se evaluó el ambiente lumínico en forma indirecta mediante fotografías hemisféricas del dosel superior tomadas a la altura del eje principal marcado de cada planta. La cámara digital (Nikon Coolpix 950) estaba provista de un lente "ojo de pez" y sujeta a un trípode. Todas las fotos fueron tomadas en cada parcela en un día nublado, con igual orientación respecto del norte magnético y niveladas horizontalmente mediante un nivel de burbuja. Se estimó el valor de luz incidente sobre cada planta empleando el programa Gap Analyzer (Frazer et al., 1999).

\section{Fenología}

El estudio fenológico de las plantas marcadas sólo se realizó en las parcelas I y II. Entre noviembre de 2011 y abril de 2013 se realizaron observaciones periódicas de la fase fenológica en la cual se encontraba el brote anual distal del eje principal de cada planta y se midió la longitud de ese brote. La frecuencia de observación y medición fue mensual de agosto a marzo durante las temporadas de crecimiento 2011-12 y 2012-13. Se definieron las siguientes fases fenológicas: 1) reposo, sin órganos nuevos ni variación de longitud entre mediciones, 2) brotación, agrandamiento notable del tamaño de las yemas e inicio del alargamiento de nudos y entrenudos del brote anual y 3) crecimiento, alargamiento de nuevos nudos y entrenudos del brote anual. Solo se observaron estructuras reproductivas en siete individuos de $R$. rubiginosa, cuatro de $M$. oblongum, tres de $P$. avium, dos de $B$. microphylla y uno de J. communis.

\section{Análisis de datos}

Las relaciones entre: altura de la planta al final del estudio, longitud de los brotes anuales producidos en la estación de crecimiento 20132014, variación en altura en el período 2012-2014, DV y radiación incidente, se evaluaron mediante correlaciones de rangos de Spearman debido a que las distribuciones de las variables estudiadas se desviaron significativamente de la normal (aún luego de aplicar transformaciones de los datos). Se graficaron las relaciones entre (1) la altura de las plantas y el diámetro basal del eje principal al final del período de crecimiento (2014), (2) la altura de la planta al final del período de crecimiento 2013 2014 y la variación en altura entre 2012 y 2014 , (3) la longitud del brote anual más distal del eje principal y la DV, y (4) la variación en altura entre 2012 y 2014 y la DV. La longitud del brote anual y DV se graficaron en escala logarítmica para mejorar su visualización.

\section{Resultados}

\section{Tamaño, crecimiento y radiación incidente}

Al final del período de estudio las plantas tenían una altura de entre $10 \mathrm{~cm}$ y $380 \mathrm{~cm}$, y un diámetro basal de entre $1,0 \mathrm{~cm}$ y $6,8 \mathrm{~cm}$ (Fig. 3A). La altura y el diámetro basal se relacionaron estrechamente. Rosa rubiginosa se diferenció claramente de las restantes especies por la mayor longitud de los brotes para un diámetro determinado (Fig. 3A). La variación de altura en el período 2012-2014 fue muy dispar e incluyó valores negativos (decrecimiento en altura) para varios individuos de B. microphylla, $R$. rubiginosa, S. patagonicus, L. hirsuta y P. avium, y en un individuo de $M$. oblongum, M. boaria, $A$. chilensis y J. communis (Fig. 3B). Pseudotsuga menziesii fue la única especie en la cual la totalidad de los individuos marcados incrementaron su altura en el período de estudio. Considerando todas las plantas, la variación en altura en el periodo 20122014 se correlacionó positivamente con la altura alcanzada por la planta en 2014 (Fig. 3B) y con la longitud del brote anual desarrollado en el período 2013-2014 (Tabla 3).

La longitud del brote distal del eje principal varió entre pocos milímetros y más de $70 \mathrm{~cm}$ (Fig. 3C). En promedio, los brotes de mayor longitud fueron los desarrollados por P. menziesii y los de menor longitud los de P. avium y B. microphylla. Dos individuos de L. hirsuta y uno de S. patagonicus no desarrollaron brote anual en el período de muestreo, de modo que no presentaron crecimiento longitudinal del eje principal, a pesar de que ese eje no mostraba signos de necrosis y conservaba hojas verdes y yemas axilares. La DV presentó notables variaciones inter- e intra-específicas (Fig. 3C). Su 
Bol. Soc. Argent. Bot. 52 (3) 2017
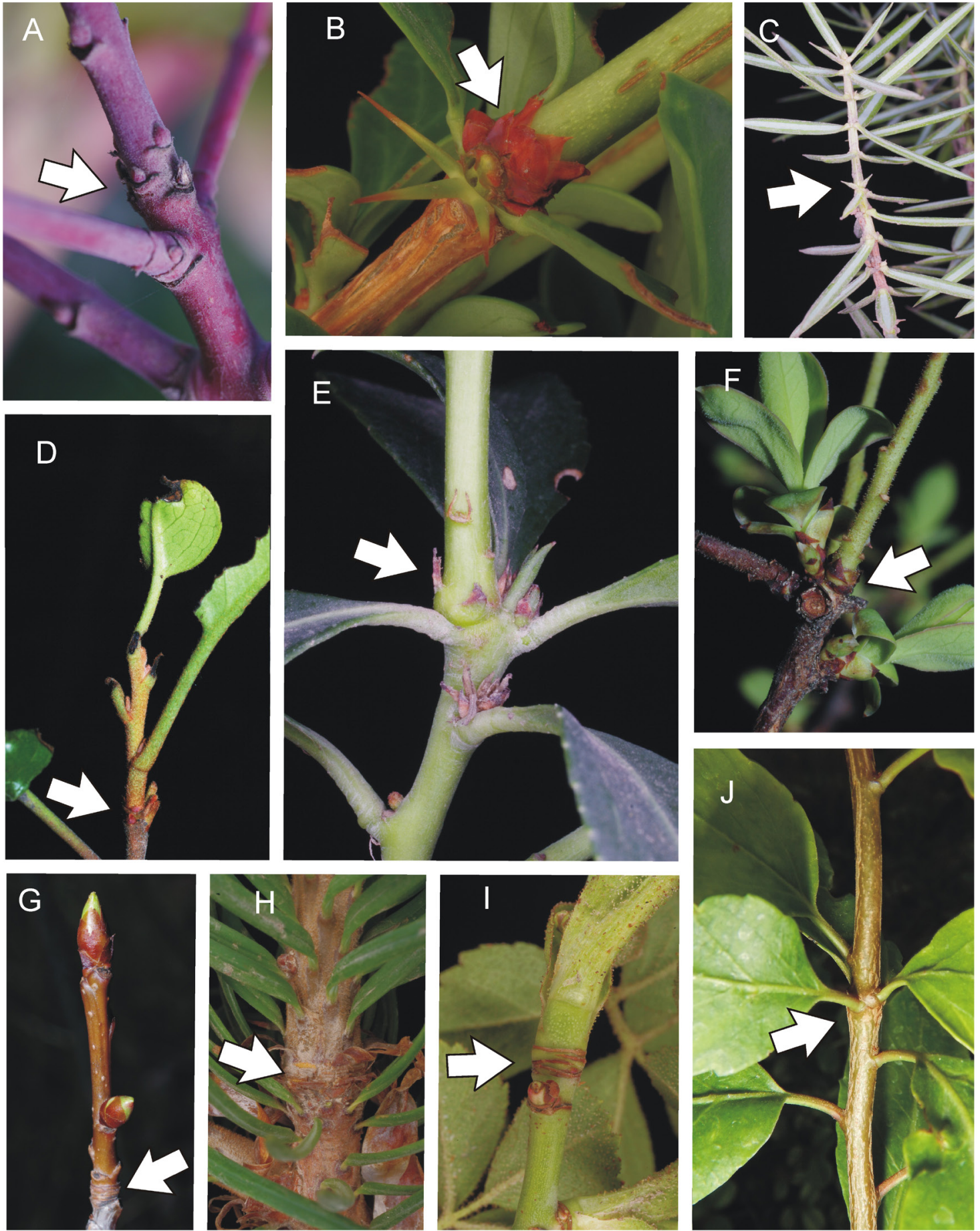

Fig. 2. Fotografías de los límites entre brotes anuales (flechas blancas) para cada especie. A. Aristotelia chilensis. B. Berberis microphylla. C. Juniperus communis. D. Lomatia hirsuta. E. Maytenus boaria. F. Myoschilos oblongum. G. Prunus avium. H. Pseudotsuga menziesii. I. Rosa rubiginosa. J. Schinus patagonicus. Nótese la presencia de entrenudos cortos, catafilos o las cicatrices dejadas por éstos sobre el tallo. 

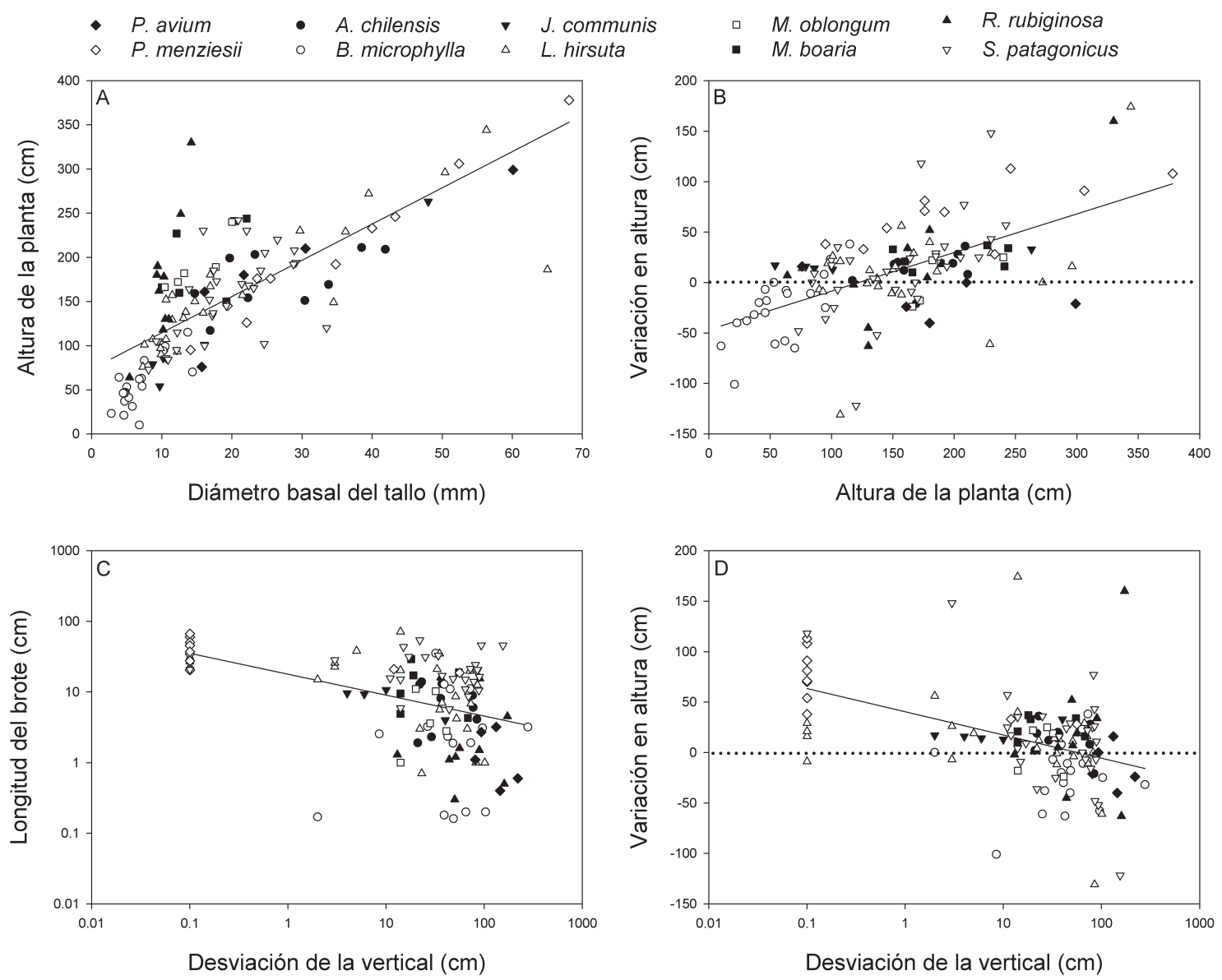

Fig. 3. Relación entre (A) la altura de las plantas y el diámetro basal del eje principal al final del período de crecimiento (2014), (B) la altura de la planta al final del período de crecimiento (2014) y la variación en altura entre 2012 y 2014, (C) la longitud del brote anual más distal del eje principal y la desviación de ese eje de la vertical (DV) y (D) la variación en altura entre 2012 y 2014 y la DV, para cada una de las plantas marcadas. Las variables longitud del brote anual y DV se representan en escala logarítmica. Se muestran las líneas de tendencia de los datos.

valor fue muy bajo o nulo en la mayoría de los individuos de P. menziesii y $J$. communis; sólo en un individuo de cada una de estas especies la DV fue mayor debido a que el tronco fue impactado por la caída de ramas de cipreses del dosel durante una tormenta de viento. En comparación con estas dos especies, la DV fue mayor en $A$. chilensis, $L$. hirsuta, M. boaria, M. oblongum y $S$. patagonicus y, en especial, en B. microphylla, $R$. rubiginosa y $P$. avium. Al considerar a la totalidad de las plantas, la DV se correlacionó negativamente con la longitud del brote distal desarrollado por el tronco y con la variación en altura (Fig. 3C, D; Tabla 3).
La radiación incidente estimada fue menor al $33 \%$ para todas las plantas marcadas; su mediana fue del $11 \%$. Excepto para B. microphylla y S. patagonicus, más de la mitad de los individuos marcados de cada especie se desarrollaron con niveles de radiación incidente mayores al $14 \%$. Al combinar los datos de todas las parcelas y especies, la radiación incidente estuvo negativamente correlacionada con la altura de la planta pero no se correlacionó con sus variaciones en altura durante este estudio o con la longitud del brote del eje principal (Tabla 3).

Entre las plantas incluidas en este estudio, sólo aquellas correspondientes a $B$. microphylla 
Tabla 3. Coeficientes de correlación de Spearman y sus niveles de significación entre las variables: altura de la planta, desviación del eje principal de la vertical (DV), longitud del brote anual del eje principal (long. brote), variación en altura de la planta y radiación incidente, considerando la totalidad de las plantas leñosas de sotobosque $(N=130){ }^{* * *}: p<0,001,{ }^{*}: p<0,05$, ns: $p \geq 0,05$.

\begin{tabular}{|lccccc|}
\hline & Radiación & Altura & DV & Long. brote & Var. altura \\
\hline Radiación & 1 & $-0,187^{*}$ & $0,061 \mathrm{~ns}$ & $0,114 \mathrm{Ns}$ & $-0,165 \mathrm{~ns}$ \\
Altura & $-\mathbf{0 , 1 8 7 *}$ & 1 & $0,124 \mathrm{~ns}$ & $0,124 \mathrm{Ns}$ & $\mathbf{0 , 4 9 9} * * *$ \\
DV & $0,061 \mathrm{~ns}$ & $0,124 \mathrm{~ns}$ & 1 & $\mathbf{- 0 , 4 2 4} * * *$ & $-\mathbf{0 , 4 1 5} * * *$ \\
Long. brote & $0,114 \mathrm{~ns}$ & $0,124 \mathrm{~ns}$ & $\mathbf{- 0 , 4 2 4} * * *$ & 1 & $\mathbf{0 , 4 4 3} * * *$ \\
Var. altura & $-0,165 \mathrm{~ns}$ & $\mathbf{0 , 4 9 9 * * *}$ & $\mathbf{- 0 , 4 1 5 * * *}$ & $\mathbf{0 , 4 4 3} * * *$ & 1 \\
\hline
\end{tabular}

desarrollaron ramas a partir de la mayoría de los nudos de los brotes anuales en crecimiento (ramas inmediatas; Barthélémy \& Caraglio, 2007); todas esas ramas constituyeron braquiblastos con número de nomofilos poco variable (entre 3 y 6 nomofilos por braquiblasto). En el caso de J. communis se desarrollaron ramas inmediatas en dos a cuatro nudos del brote anual de tres de las plantas medidas. En las restantes especies no se desarrollaron ramas inmediatas en los brotes anuales medidos.

\section{Fenología}

Considerando al conjunto de las plantas marcadas en las parcelas I y II, el período de crecimiento comprendió desde agosto hasta febrero (Fig. 4A). La brotación fue máxima entre los meses de agosto y octubre, en tanto que el mayor alargamiento de los brotes anuales se produjo entre octubre y diciembre (Fig. 4A). Las brotaciones de $R$. rubiginosa, $P$. avium, $M$. oblongum y $J$. communis se iniciaron entre agosto y septiembre (Fig. 4B). En M. oblongum, la brotación se produjo al final del período de floración. El inicio del crecimiento fue en septiembre para $A$. chilensis y entre octubre y noviembre para $P$. menziesii, M. boaria y L. hirsuta (Fig. 4B). En el caso de S. patagonicus, la brotación de las plantas marcadas presentó amplia dispersión a lo largo de la estación de crecimiento, abarcando desde agosto hasta noviembre.

La duración del crecimiento de los brotes de las parcelas I y II varió entre 1 y 8 meses. Lomatia hirsuta fue la especie con menor duración del crecimiento: hasta 1 mes en todos los individuos marcados excepto uno (con duración de 2 meses); en dos individuos no se registró crecimiento primario en el período de estudio. La duración del crecimiento en todos los individuos de $P$. avium fue de 2 meses. En las restantes especies la duración del crecimiento mostró los siguientes rangos de variación (Fig. 4B): 3-4 meses en A. chilensis y $P$. menziesii, 3-5 meses en $M$. oblongum y $R$. rubiginosa, 1-3 meses en $M$. boaria, 1-4 meses en $S$. patagonicus y 4-8 meses en J. communis.

\section{Discusión}

Patrones arquitecturales en el sotobosque

En este estudio se encontró que el crecimiento longitudinal del eje principal no necesariamente se tradujo en un incremento proporcional en la altura de las plantas, algo no observado en estudios previos sobre la arquitectura de especies arbóreas patagónicas (Puntieri et al., 1998; Barthélémy et al., 1999; Grosfeld, 2002; Stecconi, 2006; Torres et al., 2009, Magnin et al., 2014). Para la mayoría de las especies estudiadas aquí, el eje principal de los individuos de sotobosque tiende a curvarse desviándose de la vertical conforme aumenta el número de brotes anuales que se adicionan en su extremo distal (Niklas, 2007). Esta curvatura resultó en desviaciones del eje principal respecto de la 

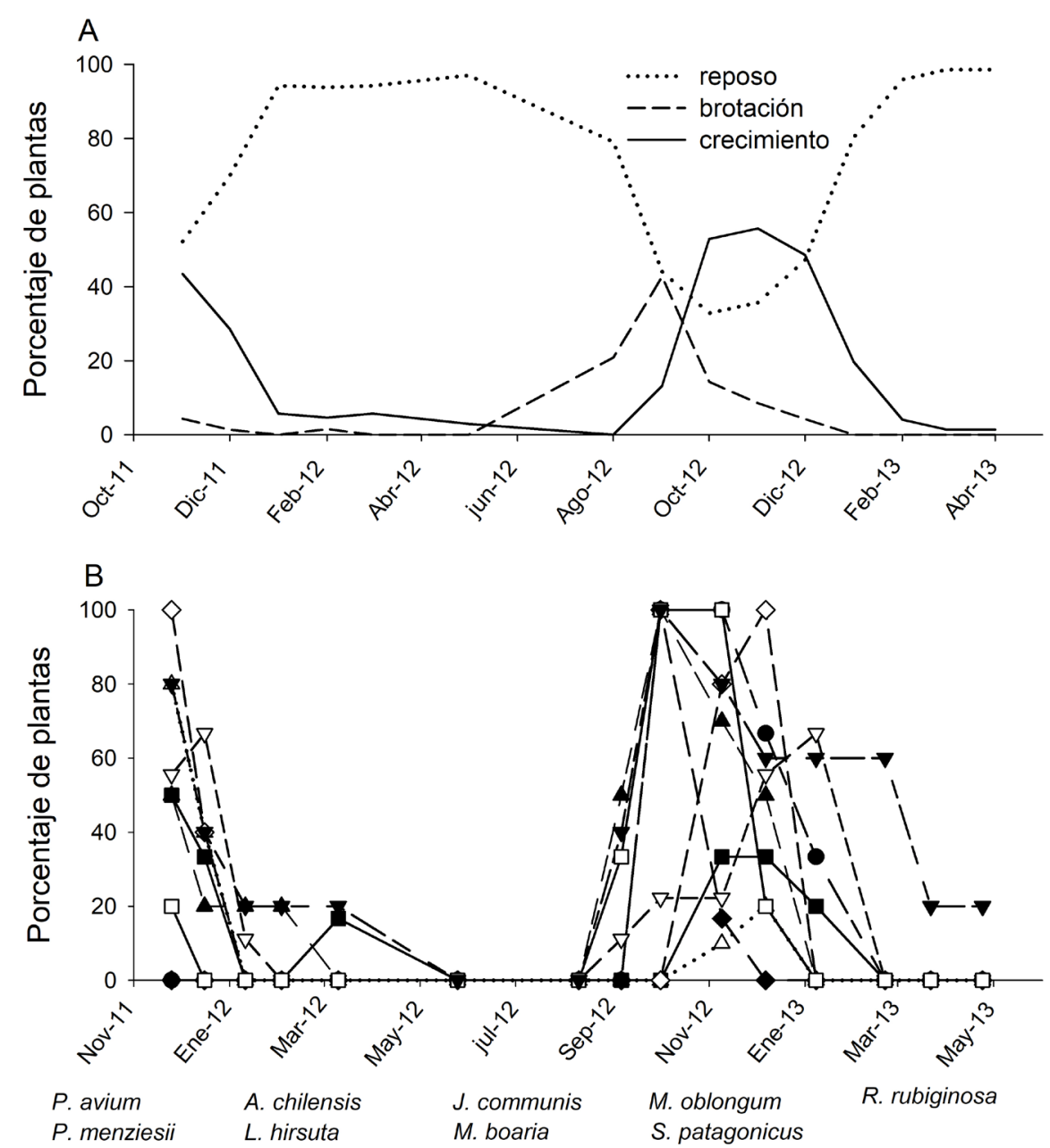

Fig. 4. (A) Períodos de reposo, brotación y crecimiento primario del conjunto de plantas leñosas, y (B) período de crecimiento primario de cada una de las especies leñosas del sotobosque de ciprés (parcelas I y II) para el período noviembre 2011 - abril 2013.

vertical (DV). De esta manera, entre las especies leñosas del sotobosque de cipresales predomina un bajo nivel de diferenciación del tronco (es decir alta DV) por sobre un nivel de diferenciación elevado (baja DV). Esto implica que tanto las especies que comúnmente presentan desarrollo arbustivo $(M$. oblongum, B. microphylla y $R$. rubiginosa) como aquellas capaces de desarrollarse como árboles $(A$. chilensis, L. hirsuta, M. boaria y S. patagonicus) no podrían alcanzar el dosel en caso de mantenerse las condiciones ambientales actuales. En este sentido, las correlaciones negativas entre la DV y la longitud del brote del eje principal y entre la DV y la variación en altura de la planta indican que la curvatura del eje principal va acompañada de disminuciones en el crecimiento longitudinal de ese eje y en la altura de la planta.

Entre las especies con bajo nivel de diferenciación del tronco se registran variaciones funcionales asociadas al patrón de crecimiento. Numerosas observaciones realizadas en el transcurso de este estudio permitieron verificar el inicio del desarrollo de brotes vigorosos a partir de porciones de tallo cercanas al nivel del suelo ("complejos reiterados" sensu Barthélémy \& Caraglio, 2007) en una alta proporción de individuos de varias de las especies 
estudiadas aquí (Tabla 2). Este tipo de brotación sugiere que, en estas plantas, el eje principal es reemplazado por otro luego de varios años o décadas de crecimiento (Fig. 5A; Ishii et al., 2007). El período de tiempo durante el cual un eje puede sostener el crecimiento vertical sin curvarse y los sitios a partir de los cuales se inician los complejos reiterados de reemplazo podrían ser fundamentales en las relaciones de dominancia y supresión del sotobosque y deberían investigarse con mayor detalle. En contraste, en P. avium no se observó el reemplazo del eje principal por complejos reiterados verticales (Fig. 5B). Durante su crecimiento en el sotobosque, el eje principal de las plantas de $P$. avium se curva, adoptando un desarrollo horizontal que se mantiene por varios años mediante la producción de brotes muy cortos (braquiblastos de pocos centímetros de longitud) y no ramificados. Respuestas arquitecturales al sombreo similares a ésta se han registrado en especies arbóreas de los géneros Fagus y Nothofagus. Los individuos de estas especies responden a la formación de claros en el dosel curvando sus ejes horizontales y retomando el crecimiento vertical (Puntieri et al., 1999; Nicolini et al., 2000; Stecconi et al., 2010). Puede suponerse que, en el caso de que $P$. avium presentara una respuesta similar se incrementaría notablemente su importancia al producirse claros en el dosel de los cipresales.

Las especies con mayor nivel de diferenciación del tronco dentro del grupo estudiado fueron $P$. menziesii y $J$. communis. En el sotobosque, todos los individuos marcados de $P$. menziesii mantuvieron el crecimiento vertical del eje principal generado a partir del estadio de plántula, con lo cual el crecimiento primario se tradujo en un incremento proporcional en altura. Bajo estas condiciones, los datos sugieren que los individuos de $P$. menziesii podrían alcanzar el estrato superior del dosel en un tiempo aproximado de 20 años (Niklas, 2007). Los individuos adultos de $P$. menziesii producen gran cantidad de semillas viables y sus plántulas y renovales son moderadamente tolerantes a la sombra (LeMay et al., 2009), por lo cual podrían avizorarse cambios muy significativos en los bosques de ciprés invadidos por esta especie. En el caso de J. communis la diferenciación del tronco no va acompañada de crecimiento anual vigoroso, con lo cual no se podría asumir que esta especie alcance el dosel.

\section{Grupos funcionales}

El crecimiento de especies leñosas asociadas a los cipresales ha sido mayormente estudiado en relación a su significancia en el establecimiento post-disturbio de dicha especie (Gobbi \& Schlichter, 1998; Raffaele \& Veblen, 1998; Gyenge et al., 2007; Steinke et al., 2008; Núñez et al., 2009). Este trabajo muestra que las especies leñosas que constituyen el sotobosque de los cipresales, tomadas en su conjunto, exhiben notables variaciones en crecimiento longitudinal y que tales variaciones no pueden ser explicadas aludiendo al grado de apertura del dosel por encima de cada planta (Valladares \& Niinemets, 2008). Dichas variaciones brindan información de relevancia para comprender la dinámica del bosque (Valladares et al., 2002). A grandes rasgos, en este estudio pudieron diferenciarse cuatro grupos funcionales que se caracterizan por: (1) ejes principales diferenciados durante un período de corta duración, reemplazados por complejos reiterados basales (Fig. 5A); (2) sistemas de ejes en los que el tronco se encuentra progresivamente menos diferenciado por pérdida de verticalidad, con producción de braquiblastos y sin reiteraciones (Fig. 5B); (3) ejes principales diferenciados, persistentes y de gran vigor (Fig. 5C); (4) ejes principales diferenciados, persistentes y de bajo vigor (Fig. 5D). Resulta interesante destacar que los últimos tres grupos funcionales corresponden a tres especies exóticas, es decir que son novedosos para los cipresales patagónicos. El incremento de la abundancia de los últimos grupos funcionales podría resultar en cambios en las tendencias sucesionales de estos bosques.

\section{Fenología y crecimiento primario}

El crecimiento primario de las plantas leñosas en el sotobosque de cipresales se produciría dentro de un período de más de seis meses que abarca desde la segunda mitad del invierno (agosto), hasta la segunda mitad del verano (febrero). El crecimiento temprano (de agosto a noviembre-diciembre) de $R$. rubiginosa, $M$. oblongum y $P$. avium se visualiza claramente por el carácter caducifolio de las mismas y los catafilos de sus yemas, que dejan cicatrices en la superficie del tallo. Es probable que $J$. communis también inicie su crecimiento tempranamente aunque, al tratarse de una especie perennifolia y con yemas desnudas, resulta menos 
A

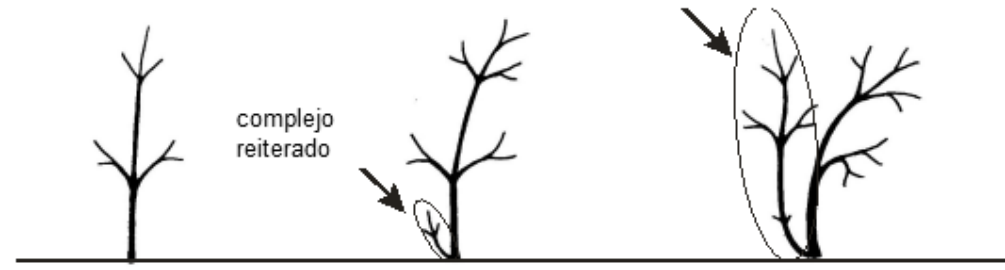

B

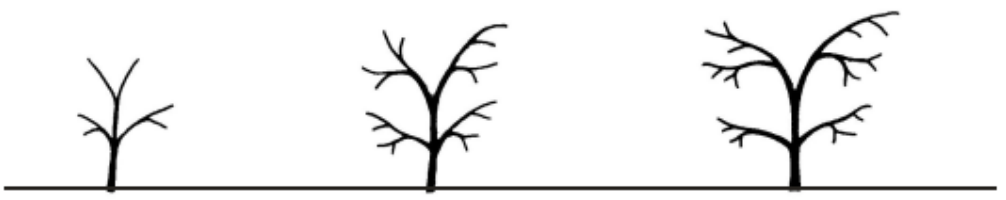

C

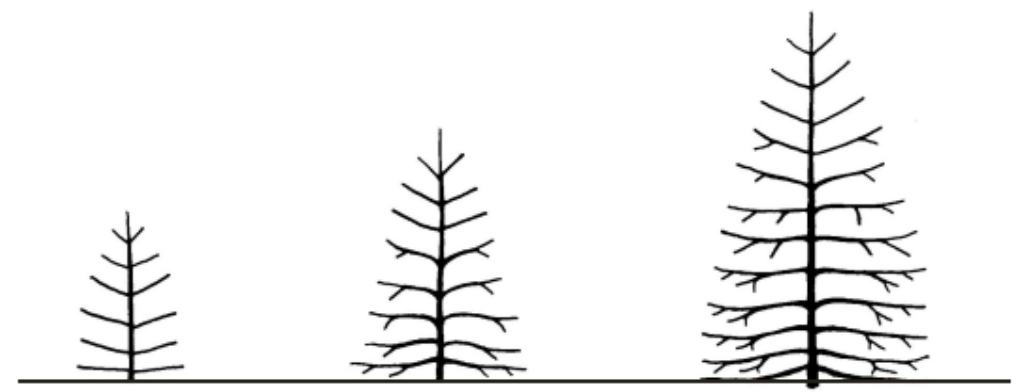

D

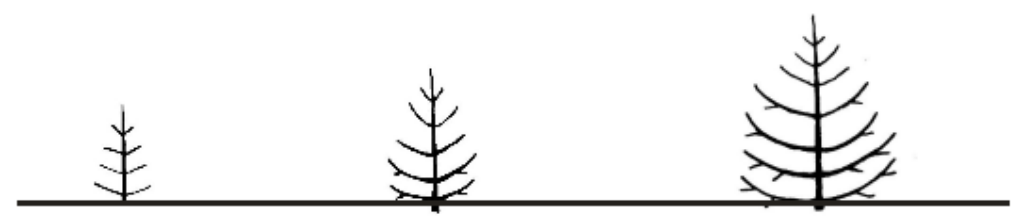

Fig. 5. Esquemas simplificados de cuatro patrones de desarrollo del eje principal que definen a cuatro grupos funcionales de las plantas leñosas de sotobosque: (A) con formación de complejos reiterados que relevan el eje principal (flechas) luego de la curvatura y pérdida de vigor de éste a lo largo de varios años (e.g. Aristotelia chilensis), (B) con eje principal horizontal y formado por braquiblastos, en "forma de espera" y sin reiteraciones (e.g. Prunus avium), (C) sostenidamente diferenciado y vertical de gran crecimiento anual, sin reiteraciones (e.g. Pseudotsuga menziesii) y (D) sostenidamente jerárquica y vertical de escaso crecimiento anual (e.g. Juniperus communis), sin reiteraciones. Los tres esquemas que se muestran en orden horizontal corresponden a distintas etapas de desarrollo de la misma planta.

precisa la identificación de su momento de brotación estacional. Como en otras especies de Cupressaceae, el crecimiento de $J$. communis podría ser de tipo continuo (Grosfeld, 2002), lo cual es consistente con la gran variación encontrada aquí en su fenología para una misma estación y en el mismo sitio.
Las especies nativas A. chilensis, M. boaria, L. hirsuta y S. patagonicus crecieron en longitud principalmente en primavera, aunque con notables variaciones intra-específicas en las tres últimas especies. Se destaca el hecho de que no se registró crecimiento primario en el eje principal en todo el 
transcurso de este estudio para dos individuos de L. hirsuta y uno de S. patagonicus. La ausencia de crecimiento primario en un eje podría significar la muerte del mismo una vez que sus hojas se deshidrataran, algo que merece ser investigado.

En el caso de $P$. menziesii, el crecimiento de los brotes del tronco tuvo lugar entre mediados y fines de primavera, es decir que fue relativamente tardío y breve, a pesar de que los individuos marcados de esta especie fueron los que desarrollaron brotes de mayor tamaño y los que más incrementaron su altura en el período abarcado por este estudio. En comparaciones intra-específicas previas se demostró que la duración del crecimiento primario se vincula positivamente con el tamaño de los brotes formados (Puntieri et al., 1998; Costes \& GarcíaVillanueva, 2007), pero la relación entre duración del crecimiento y tamaño de los brotes se debilita cuando el nivel de variación inter-específica en las tasas de crecimiento primario es elevado (James \& Drenovsky, 2007).

Se registró cierto grado de solapamiento fenológico inter-específico: hacia la segunda mitad de la primavera (en noviembre) la mayoría de los individuos de la totalidad de las especies se encontraban en crecimiento, en tanto que en los meses de verano el crecimiento fue prácticamente nulo. Estudios previos en árboles caducifolios han propuesto que las plantas de sotobosque se benefician al desarrollar hojas con anticipación a la brotación de los árboles (Valladares \& Niinemets, 2008; Richardson \& O'Keefe, 2009). En el presente caso, tratándose de un bosque perennifolio, la anticipación de la brotación podría interpretarse como ventajosa en la competencia por la luz entre las especies del sotobosque. En la región norpatagónica de la Argentina, donde predominan las lluvias en otoño-invierno y el estrés por falta de agua se hace frecuente en el período estival (Barros et al., 1983; Conti, 1998), las presiones de selección natural favorecerían a aquellas especies que exhiben su crecimiento estacional entre fines de invierno y primavera temprana (como $R$. rubiginosa, $M$. oblongum, $P$. avium y $J$. communis). Es destacable que la mayoría de estas especies son exóticas invasoras en Patagonia (Schüttler \& Karez, 2009), lo cual indicaría que el uso espacio-temporal atípico que las caracteriza podría ser uno de los componentes de su éxito (Lockwood et al., 2007; Wolkovich \& Cleland, 2011; Lediuk et al., 2014, 2016).

\section{Conclusiones}

El periodo y la magnitud del crecimiento longitudinal del eje principal de las plantas leñosas no necesariamente reflejan el incremento en altura de esas plantas. En las especies que habitan los sotobosques de cipresales se pudieron identificar cuatro grupos funcionales según el grado de diferenciación y persistencia del eje principal, que determinan las posibilidades de alcanzar el dosel superior. Es común que los ejes principales de las plantas leñosas nativas de sotobosque se desvíen de la verticalidad y que ocurra su reemplazo a partir de complejos reiterados basales. Este grupo funcional se desarrolla en el sotobosque con pocas posibilidades de alcanzar el dosel. Algunas de las especies exóticas presentes en estos bosques exhiben patrones de desarrollo que ameritan su ubicación en diferentes grupos funcionales, uno de los cuales se caracteriza por mantener la diferenciación y crecimiento del eje principal en el sotobosque, con altas posibilidades de alcanzar el dosel. La presencia de las especies exóticas en los cipresales puede ser favorecida por el desarrollo de grupos funcionales asociados a patrones arquitecturales y/o fenológicos novedosos en relación a los que exhiben las especies nativas con las que coexisten.

\section{Agradecimientos}

Al INTA EEA Bariloche por permitirnos acceder a las parcelas I y II ubicadas en Las Golondrinas y Mallín Ahogado. Al personal y dueño de la Estancia Siete Cóndores por permitirnos ingresar a las parcelas III y IV ubicadas en Confluencia Traful. Este trabajo fue realizado con la financiación de la Universidad Nacional del Comahue, proyecto $\mathrm{N}^{\circ}$ B164.

\section{Bibliografía}

AGUIAR, M. \& O. SALA 1994. Competition, facilitation, seed distribution and the origin of patches in a Patagonian steppe. Oikos 70: 26-34.

AMOROSO, M., L.D. DANIELS \& B.C. LARSON. 2012. Temporal patterns of radial growth in 
declining Austrocedrus chilensis forests in Northern Patagonia: The use of tree-rings as an indicator of forest decline. For. Ecol. Manage. 265: 62-70.

BARROS, V.K., V.H. CARDON, C.L. MOYANO, R.J. MENDEZ, J.C. FORQUERA \& O. PIZZIO. 1983. Cartas de precipitación zona oeste de la provincia de Río Negro y Neuquén. Universidad Nacional del Comahue, Bariloche.

BARTHÉLÉMY, D. \& Y. CARAGLIO. 2007. Plant architectural: a dynamic, multilevel and comprehensive approach to plant form, structure and ontogeny. Ann. Bot. 99: 375-407.

BARTHÉLÉMY, D., J. GROSFELD, F. BOUROULETHALLARD \& C. DUCATILLION. 1999. Biología, crecimiento y desarrollo. En: E. Tessier du Cross (ed.), Los Cipreses: un manual práctico, pp. 27-33. Studio Leonardo, Florencia.

BRAN, D., A. PÉREZ, D. BARRIOS, M.J. PASTORINO \& J. AYESA. 2002. Eco-región Valdiviana: distribución actual de los bosques de "Ciprés de la Cordillera" (Austrocedrus chilensis)- Escala 1:250.000. INTA-Adm. Parques NacionalesFundación Vida Silvestre Argentina, Bariloche.

CANHAM, L. 1988. Growth and canopy architecture of shade-tolerant trees: response to canopy gaps. Ecology 69: 786-795.

CARABELLI, F.A., I.A. ORELLANA, M.M. JARAMILLO \& M.F. GÓMEZ. 2006. Modification of the flora composition in edges of fragmented Austrocedrus chilensis forests in Patagonia. Invest. Agrar.: Sist. Recur. For. 15: 42-49.

CONNELL, J.H. \& R.O. SLATYER. 1977. Mechanism of succession in natural communities and their role in community stability and organization. Am. Nat. 111 (982): 1119-1144.

CONTI, H.A. 1998. Características climáticas de la Patagonia. En: Correa M. (ed.), Flora Patagónica I, pp. 31-47, Colección científica del INTA, Buenos Aires.

CORNELISSEN J.H.C., S. LAVOREL, E. GARNIER, S. DÍAZ, N. BUCHMANN, D.E. GURVICH, P.B. REICH, H. TER STEEGE, H.D. MORGAN, M.G.A. van der HEIJDEN, J.G. PAUSAS \& H. POORTER. 2003. A handbook of protocols for standardized and easy measurement of plant functional traits worldwide. Aust. J. Bot. 51: 335380 .

COSTES, E. \& E. GARCÍA-VILLANUEVA. 2007. Clarifying the effects of dwarfing rootstock on vegetative and reproductive growth during tree development: a study on apple trees. Ann. Bot. 100: 347-357.

DEZZOTTI, A. \& L. SANCHOLUZ. 1991. Los bosques de Austrocedrus chilensis en Argentina: ubicación, estructura y crecimiento. Bosque 12: 43-52.
DÍAZ, S., M. CABIDO, M. ZAK, E. MARTÍNEZ CARRETERO \& J. ARANÍBAR. 1999. Plant functional traits, ecosystem structure and land-use history along a climatic gradient in central-western Argentina. J. Veg. Sci. 10: 651-660.

DÍAZ, S., J. KATTAGE, J.H. CORNELISSEN, I.J. WRIGHT, S. LOVOVEL, S. DRAY, B. REU, M. KLEYER, C. WIRTH, E. GARNIER, G. BÖNISCH, M. WESTOBY, H. POORTER, P.B. REICH, A.T. MOLES, J. DICKIE, A.N. GILLISON, A.E. ZANNE, J. CHAVE, S.J. WRIGHT, S.N. SHEREMET'EV, H. JACTEL, C. BARALOTO, B. CERABOLINI, S. PIERCE, B. SHIPLEY, D. KIRKUP, F. CASANOVES, J.S. JOSWIG, A. GÜNTHER, V. FALCZUK, N. RÜGER, M.D. MAHECHA \& L.D. GORNÉ. 2016. The global spectrum of plant form and function. Nature 529: 167-171.

DIMITRI, M.J. 1978. Enciclopedia argentina de agricultura y jardinería. Editorial ACME, Buenos Aires.

DONOSO, C. 2006. Las especies arbóreas de los bosques templados de Chile y Argentina. Autoecología. Marisa Cuneo Ediciones, Valdivia.

EDELIN, C. 1991. Nouvelles donnée sur l'architecture des arbres sympodiaux: le concept de plan d'organization. In: C. Edelin (Ed.), L'Arbre: Biologie et Développement 127-154. Naturalia Monspeliensia, $2^{\text {nd }}$ International Tree Conference.

FORD, E. D. 2014. The dynamic relationship between plant architecture and competition. Front. Plant Sci. 5: $1-13$.

FRAZER, G.W., C.D. CANHAM, \& K. P. LERTZMAN. 1999. Gap Light Analyzer (GLA), Version 2.0: Imaging software to extract canopy structure and gap light transmission indices from true-color fisheye photographs. Copyright 1999: Simon Fraser University, Burnaby, BC, and the Institute of Ecosystem Studies, Millbrook, New York. http:// www.rem.sfu.ca/forestry/ index.htm or http://www. ecostudies.org.

GIVINISH, T.J. 1988. Adaptation to sun and shade, a whole plant perspective. Aust. J. Physiol. 15: 63-92.

GOBBI, M. \& T. SCHLICHTER. 1998. Survival of Austrocedrus chilensis in relation to microsite conditions and forest thinning. For. Ecol. Manage. 111: 137-146.

GROSFELD, J. 2002. Análisis de la variabilidad morfológica y arquitectural de Austrocedrus chilensis, Fitzroya cupressoides, Pilgerodendron uviferum y Cupressus sempervirens L. (Cupressaceae). Tesis Doctoral, Universidad Nacional del Comahue, Bariloche.

GYENGE, J.E., M.E. FERNÁNDEZ \& T. SCHLICHTER. 2007. Influence of radiation and drought on gas exchange of Austrocedrus chilensis seedlings. Bosque 28: 220-225. 
HALLÉ, F., R.A. OLDEMAN \& P.B. TOMLINSON. 1978. Tropical trees and forest. An architectural analysis. Springer, Berlin.

HENRÍQUEZ, J.M. \& C.H. LUSK. 2005. Facilitation of Nothofagus antarctica (Fagaceae) seedlings by the prostrate shrub Empetrum rubrum (Empetraceae) on glacial moraines in Patagonia. Austral Ecol. 30: 877-882.

HÉRAULT, B., B. BACHELOT, L. POORTER, V. ROSSI, F. BONGERS, J. CHAVE, T. PAINE, F. WAGNER, C. BARALOTO. 2011. Functional traits shape ontogenetic growth trajectories of rain forest tree species. J. Ecol. 99: 1431-1440.

ISHII, H.T., E.D. FORD \& M.C. KENNEDY. 2007. Physiological and ecological implications of adaptive reiteration as a mechanism for crown maintenance and longevity. Tree Physiol. 27: 455-462.

JAMES, J.J. \& R.E. DRENOVSKY. 2007. A basis for relative growth rate differences between native and invasive forb seedlings. Rangeland Ecol. Manage. 60: 395-400.

KITZBERGER, T., T.T. VEBLEN \& R. VILLALBA. 1997. Climatic influences on fire regimes along rainforest-to-xeric woodland gradient in northern Patagonia. J. Biogeogr. 24: 35-47.

KITZBERGER, T., D.F. STEINAKER \& T.T. VEBLEN. 2000. Effects of climatic variability on facilitation of tree establishment in northern Patagonia. Ecology 81: 1914-1924.

LARADE, S.E. \& E.W. BORK. 2011. Aspen forest overstory relations to understory production. Can. J. Plant Sci. 91: 847-851.

LEDIUK, K., M.A. DAMASCOS, J. PUNTIERI \& M. SVRIZ. 2014. Differences in phenology and fruit characteristic between invasive and native woody species favor exotic species invasiveness. Plant Ecol. 215: 1455-1467.

LEDIUK, K., M.A. DAMASCOS, J. PUNTIERI \& M. I. DE TORRES CURTH. 2016. Population dynamics of an invasive tree, Sorbus aucuparia, in the understory of a Patagonian forest. Plant Ecol. 217: 899-911.

LEMAY, V., A. POMMERENING \& P. MARSHALL. 2009. Spatio-temporal structure of multi-storied, multi-aged interior Douglas fir (Pseudotsuga menziesii var. glauca) stands. J. Ecol. 97: 1062-1074.

LETOURNEAU, F. J. 2006. Estudio de las interacciones positivas y negativas sobre el crecimiento de Austrocedrus chilensis durante una etapa inicial de desarrollo, en un matorral sucesional mésico. Universidad Nacional del Comahue, Bariloche.

LETOURNEAU, F. J., E. ANDENMATTEN \& T. SCHLICHTER. 2004. Effect of climatic conditions and tree size on Austrocedrus chilensis-shrub interactions in northern Patagonia. For. Ecol. Manage. 191: 29-38.
LETOURNEAU, F.J., BASIL, J.G. \& P. KLASMER. 2016. Serie de Datos de Precipitaciones Golondrinas, 1953-2011. Comunicaciones Técnicas $\mathrm{N}^{\circ} 28$, Agrometeorología. EEA Bariloche, INTA.

LOCKWOOD, J., M. HOOPES, M. MARCHETTI. 2007. Invasion ecology. Blackwell Publishing, Oxford.

MAGNIN A., J. PUNTIERI J. \& R. VILLALBA. 2014. Interannual variations in primary and secondary growth of Nothofagus pumilio and their relationships with climate. Trees Struct. Funct. 28: 1463-1471.

MILLET, J., A. BOUCHARD \& C. EDELIN. 1998. Plant succession and tree architecture: an attempt at reconciling two scales of analysis of vegetation dynamics. Acta Biotheor. 46: 1-22.

MORELlO, J., MATTEUCCI, S.D., RODRÍGUEZ, A.F., SILVA, M.E. 2012. Ecorregiones y complejos ecosistémicos argentinos. Orientación Gráfica Editora, Buenos Aires.

MUELLER-DOMBOIS, D. \& H. ELLENBERG. 1974. Aims and methods of vegetation ecology. Wiley, New York.

NAGASHIMA, H. \& K. HIKOSAKA. 2011. Plants in a crowded stand regulate their height growth so as to maintain similar heights to neighbours even when they have potential advantages in height growth. Ann. Bot. 108: 207-214.

NICOLINI, E., D. BARTHÉLÉMY \& P. HEURET. 2000. Influence de la densité du couvert forestier sur le développement architectural de jeunes chênes sessiles, Quercus petraea (Matt.) Liebl. (Fagaceae), en régénération forestière. Can. J. Bot. 78: 15311544.

NIKLAS, K.J. 2007. Maximum plant height and the biophysical factors that limit it. Tree Physiol. 27: 433-440.

NÚÑEZ, C., E. RAFFAELE, M. A. NÚÑEZ \& F. CUASSOLO. 2009. When do nurse plants stop nursing? Temporal changes in water stress levels in Austrocedrus chilensis growing within and outside shrubs. J. Veg. Sci. 20: 1064-1071.

PASTORINO, M.J. \& L.A. GALLO. 2009. Preliminary operational genetic management units of a highly fragmented forest tree species of southern South America. For. Ecol. Manage. 257: 2350-2358.

PÉREZ-HARGUINDEGUY, N. S. DÍAZ, E. GARNIER , S. LAVOREL, H. POORTER, P. JAUREGUIBERRY, M. S. BRET-HARTE, W. K. CORNWELL, J. M. CRAINE, D. E. GURVICH, C. URCELAY, E. J. VENEKLAAS, P. B. REICH, L. POORTER, I. J. WRIGHT, P. RAY, L. ENRICO, J. G. PAUSAS, A. C. DE VOS, N. BUCHMANN, G. FUNES, F. QUÉTIER, J. G. HODGSON, K. THOMPSON, H. D. MORGAN, H. TER STEEGE, M. G. A. VAN DER HEIJDEN, L. SACK, B. 
BLONDER, P. POSCHLOD, M. V. VAIERETTI, G. CONTI, A. C. STAVER, S. AQUINO \& J. H. C. CORNELISSEN. 2013. New handbook for standardised measurement of plant functional traits worldwide. Aust. J. Bot. 61: 167-234.

POORTER, L., L. BONGERS \& F. BONGERS. 2006. Architecture of 54 moist-forest tree species: traits, trade-offs and functional groups. Ecology 87: 12891301.

PUNTIERI, J., D. BARTHÉLÉMY, P. MARTÍNEZ, E. RAFFAELE \& C. BRION. 1998. Annual shoot growth and branching patterns in Nothofagus dombeyi (Fagaceae). Can. J. Bot. 76: 673-685.

PUNTIERI, J., E. RAFFAELE, P. MARTÍNEZ, D. BARTHÉLÉMY \& C. BRION. 1999. Morphological and architectural features of young Nothofagus pumilio (Poepp. \& Endl.) Krasser (Fagaceae). Bot. J. Linn. Soc. 130: 395-410.

PUNTIERI, J.G., M.S. SOUZA, C. BRION, C. MAZZINI \& D. BARTHÉLÉMY. 2003. Axis differentiation in two south american Nothofagus species (Nothofagaceae). Ann. Bot. 92: 589-599.

RAFFAELE, E. \& T.T. VEBLEN. 1998. Facilitation by nurse shrubs of resprouting behavior in a post-fire shrubland in northern Patagonia, Argentina. J. Veg. Sci. 9: 693-698.

REICH, P.B., D.S. ELLSWORTH \& M.B. WALTERS. 1998. Leaf structure (Specific Leaf Area) modulates photosynthesis-nitrogen relations: evidence from within and across species and functional groups. Funct. Ecol. 12: 948-958.

REICH, P.B., I.J. WRIGHT, J. CAVENDER-BARES, J.M. CRAINE \& J. OLEKSYN. 2003. The evolution of plant functional variation: traits, spectra and strategies. Int. J. Plant Sci. 164 (Suppl. 3): S143-S64.

RICHARDSON, A.D. \& J. O'KEEFE. 2009. Phenological differences between understory and overstory: a case study using the long-term Harvard forest records. In: Noormets A. (ed.), Phenology of ecosystem processes, pp. 87-117. Springer, Berlin.

RODRÍGUEZ-GARCÍA, E., C. ORDÓÑEZ \& F. BRAVO. 2011. Effects of shrub and canopy cover on the relative growth rate of Pinus pinaster Ait. seedlings of different sizes. Ann. For. Sci. 68: 337-346.

SCHÜTTLER, E. \& C.S. KAREZ. 2009. Especies exóticas invasoras en las Reservas de Biosfera de América Latina y el Caribe. UNESCO, Montevideo.

SEKERCIOGLU, C. 2010. Ecosystem functions and services. In: Sodhi, N. S. \& P. R. Ehrlich (Eds.), Conservation Biology for All, pp. 45-72. Oxford Univ. Press, Oxford.

STECCONI, M. 2006. Variabilidad arquitectural de especies nativas de Nothofagus de la Patagonia ( $N$. antarctica, N. pumilio, N. dombeyi). Tesis Doctoral. CRUB, Universidad Nacional del Comahue, Bariloche
STECCONI, M., J. PUNTIERI \& D. BARTHÉLÉMY. 2010. An architectural approach of growth forms of Nothofagus pumilio (Nothofagaceae) along an altitudinal gradient. Botany 88: 699-709.

STECCONI, M., D. BARTHÉLÉMY \& J. PUNTIERI. 2014. Una mirada arquitectural a las formas de crecimiento de Nothofagus antarctica (ñire) en el norte de Patagonia. Patagonia Forestal, 20: 12-17.

STEINKE, L.R., A.C. PRÉMOLI, C.P. SOUTO \& M. HEDRÉN. 2008. Adaptive and neutral variation of the resprouter Nothofagus antarctica growing in distinct habitats in north-western Patagonia. Silva Fenn. 42: 177-188.

SVRIZ, M., M.A. DAMASCOS, H. ZIMMERMANN \& I. HENSEN. 2013. The exotic shrub Rosa rubiginosa as a nurse plant. Implications for the restoration of disturbed temperate forests in Patagonia, Argentina. For. Ecol. Manage. 289: 234-242.

TORRES, C., J. PUNTIERI \& M. STECCONI. 2009. Estudio comparativo del crecimiento primario y la ramificación de brotes anuales en dos especies de Nothofagus (Nothofagaceae) y en híbridos interespecíficos. Bol. Soc. Arg. Bot. 44: 287-303.

TORTORELLI, L.A. 2009. Maderas y bosques argentinos. 2da. edición. Orientación Gráfica Editora, Buenos Aires.

VALLADARES, F., J. SKILLMAN \& R.W. PEARCY. 2002. Convergence in light capture efficiencies among tropical forest understorey plants with contrasting crown architectures: a case of morphological compensation. Am. J. Bot. 89: 1275-84.

VALLADARES, F. \& U. NIINEMETS. 2008. Shade tolerance, a key plant feature of complex nature and consequences. Annu. Rev. Ecol. Syst. 39: 237-57.

VEBLEN, T.T. \& C.D. LORENZ. 1988. Recent vegetative changes along the forest/steppe ecotone of northern Patagonia. Ann. Assoc. Amer. Geogr. 78: 93-111.

VEBLEN, T.T., M. MERMOZ, C. MARTÍN \& E. RAMILO. 1989. Effects of exotic deer on forest regeneration and composition in northern Patagonia. J. Appl. Ecol. 26: 711-724.

VEBLEN, T.T., T. KITZBERGER \& A. LARA. 1992. Disturbance and forest dynamics along a transect from Andean rain forest to Patagonian shrubland. J. Veg. Sci. 3: 507-520.

VESTER, H.F. M. 2002. Modelos arquitectónicos en la flora arbórea de la península de Yucatán. Bol. Soc. Bot. México 71: 45-57.

VILLALBA, R. \& T.T. VEBLEN. 1998. Influences of large-scale climatic variability on episodic tree mortality in Northern Patagonia. Ecology 79: 26242640 .

WOLKOVICH, E.M. \& E.E. CLELAND. 2011. The phenology of plant invasions: a community ecology perspective. Front. Ecol. Environ. 9: 287-294. 
Bol. Soc. Argent. Bot. 52 (3) 2017

ZALAMEA, P.-C., P. HEURET, C. SARMIENTO, M. RODRÍGUEZ, A. BERTHOULY, S. GUITET, E. NICOLINI, C. DELNATTE, D. BARTHÉLÉMY \& S. R. STEVENSON. 2012. The genus Cecropia: a biological clock to estimate the age of recently disturbed areas in the Neotropics. PLoS ONE 7(8): e42643.

Recibido el 22 de agosto de 2016, aceptado el 17 de febrero de 2017. 